soap or any good superfatted soap. If the patient insists on a medicated soap order the first you think of, for I do not believe that the small amount of the drug contained is of any value, considering how short a time it remains on the skin and how much it is diluted by the large quantity of fluid with which it is brought in contact. After removing the diseased epithelium in this way some application must be rubbed thoroughly into the patches. In the case of acute psoriasis very mild remedies should be ordered, such as 2 to 4 per cent. salicylic ointment or a 5 per cent. naphthol ointment. These are usually sufficient to cause involution of the disease and effect a cure. But in the chronic forms stronger applications are necessary. Of these the best is a tar preparation, either the old and dirty unguentum picis liquidæ, or oleum cadini, or oleum fagi. These are wood-tar preparations but it is now rather the fashion to prescribe coal-tar preparations, such as pix carbonis. A drachm of liquor picis carbonis to the ounce of lanolin can be rubbed in or the same amount of oil of cade to the ounce of olive oil brushed in and then boric powder freely dusted on. The old-fashioned method of painting pure tar on to the spots and then leaving the patient in a hot bath for half an hour and washing the tar off with soft soap is excellent, inasmuch as it avoids the unpleasant after-smell of tar, which some patients complain of when they have to go to their business.

The second drug largely used is chrysarobin, but it has disadvantages. It does not always cure the disease, it does not prevent recurrence, sometimes it sets up an acute dermatitis and may provoke a general o'tbreak of psoriasis over the whole body. It stains the linen indelibly, it turns the hair yellow and the skin a purple brown, and may induce toxic symptoms of fever, pain, discomfort, and sleeplessness. It is usually rubbed in as an ointment, but if $I$ use it I usually prescribe a 10 per cent. solution in traumaticin, which is gutta-percha dissolved in chloroform. This is painted on twice a day with a brush for ten days, during which the patient does not take a bath; at the end of this period he washes the application off. This is a cleanly way of applying chrysarobin and the result is often good. Chrysarobin plasters are also a good method of using the drug.

Pyrogallol is also another substance which in 10 per cent. ointments has many advocates, but if used on large areas it easily induces toxic symptoms, of which collapse and hæmoglobinuria are the most common. For the face and scalp, ointments containing ammoniated mercury, carbolic acid, and naphthol are the best; they are cleanly and easily applied.

Small doses of $x$ rays, violet light, radium, and highfrequency currents sometimes give good results, but the great secret of the treatment of psoriasis is patience and the seeing that your instructions are carried out without any slackness on the patient's part until every patch of psoriasis has been got rid of

$\mathrm{I}$ almost hesitate to include in my paper a skin disease which is very common in hospital practice but which ought never to occur in private practice. I refer to scabies, or common itch. No doubt many practitioners have never seen a case in private practice, but while man is what he is and the possibilities of infection are what they are we sometimes see cases even among the apparently most respectable members of society. And to overlook or wrongly diagnose a case of scabies may be disastrous. When I see a general eruption, scratched or pustular, and the patient complains of itching I always look for burrows, whoever the patient. If $I$ can demonstrate an acarus there can be no question of the diagnosis, and it is not any business of mine to inquire from whom the acarus came. It is a fact, however, that one variety of mange in the dog is due to an acarus which may infect man, and persons who have much to do with dogs may become infected in this way, The face is always affected in these cases, whereas ordinary scabies does not cause any lesions of the face in adults.

The treatment of scabies aims essentially at opening up the burrows, destroying the acari, and allaying the itching. The best way is to make the patient soak in a hot bath for a quarter of an hour, then scrub himself thoroughly with soft soap, using a nail brush for the hands and feet. After washing off the soap the sulphur ointment of the pharmacopoeia is rubbed all over the skin, except the face, and left on for three days. The patient then has a starch bath to allay the irritation and applies boric ointment to any patches of dermatitis which may be present. It is important not to use any sulphur after the third day, otherwise an unpleasant degree of eczema or artificial dermatitis may be set up which will also cause great itching. In place of sulphur, $\beta$-naphthol in 20 per cent. ointment may be rubbed in after the bath and again on the three following days. It has the advantage that it is odourless and cleaner than sulphur, but it is not so efficacious. The same may be said of balsam of Peru, and I myself prefer to order sulphur. In any case, the clothes must be thoroughly disinfected and the patient kept under observation for ten days to make sure that all ova have been destroyed.

\section{A PRELIMINARY NOTE ON THE CLINICAL VALUE OF THE ANTITRYPTIC INDEX OF THE BLOOD IN TUBERCULOSIS.}

By F. L. GOLLA, M.B. Oxon., M.R.C.P. LoNd., ASSISTANT PHYSICIAN TO ST. GEORGE'S HOSPITAL.

(Irom the Physiological Laboratory of the University of London.)

THE significance of the antitryptic power of the serum in pathological conditions has recently received some attention, but the non-employment of accurate quantitative methods has been responsible for ambiguous and completely disappointing results. The method which has been followed for the most part is that devised by Müller and Jochmann. The tryptic pus obtained from empyemata and abscesses was sown in platinum loopfuls on a Löfller plate. To each loopful of pus a varying number of loopfuls of serum was added and the antitryptic efficacy of the serum judged by the relative number of drops required to inhibit a drop of the; tryptic-acting pus from digesting the underlying plate surface sufficiently to cause a visible depression. It is obvious that such a method could hardly be expected to give any but the roughest results, and that the comparison between one drop of trypsin-containing substance mixed with a certain number of times its volume of colloidal fluid and another with a different multiple of colloid could give no information as to the true inhibitory powers of the serum albumin. The criticisms of Klienberger and Schulz as to the difficulties in obtaining constant Löffler plates and of mixing and distributing uniformly the several droplets are amply justified. Wiens succeeded in an apparent increase in the anti. ferment power of the blood in cases of tuberculosis, and in agreement with Bittorf an alteration in cases of pneumonia, but his results have been traversed by Klienberger and Scholz, who failed to get any constant results by use of this method. Equally opposed to all our knowledge of the phases of enzyme activity are those adopted by Bergmann and Meyer who looked for the end-point reaction in a casein solution digested by a trypsin solution to which varying amounts of serum were added by means of Gross's method and expressed their results in - or + form. The inhibitory action of products of digestion and the decrease of available substrate tend to cause the determination of the end-points in such digestions to become indefinite. The only methods open to the investigator are such as allow him by continuous observations of the progress of the reaction to obtain accurate information as to the velocity of reaction when equal ferment strengths are allowed to act on a substrate to which equal amounts of different sera have been added. The chemical method of determining the increase of incoagulable nitrogen in samples withdrawn at intervals from the digest has to be rejected owing to the circumstances under which clinical observations are conducted. The amount of serum obtainable from a patient is too small and the method is too laborious to permit of such observations ever becoming a matter of clinical routine. The method of electrical conductivity as introduced by Victor Henri into the study of enzymes and which has been made familiar by the work of Bayliss on tryptic action offers itself as an extremely convenient one for this purpose. During a tryptic digestion the rise of electrical conductivity of the digest offers itself as a simple and accurate method of following the course of the reaction, and with extremely small quantities of serum consistent and accurate measurements of 
inhibitory powers can be obtained. This method has been adopted in the following experiments and the encouraging results that it has yielded have induced me to publish this preliminary account of observations on tuberculosis in the hope that it may attract other workers to contribute the large number of results that will be necessary before what promises to be a new chapter in pathology can be established on a firm basis. The method used for estimating the electrical conductivity of the solutions was that of Kolrausch with the rapidly alternating current and telephone.

The vessels used were after the pattern of those of Henri, having a capacity of 15 cubic centimetres. To permit of ready cleansing it is important that they should be widemouthed and stoppered to prevent evaporation. The resistance capacity of the vessels was redetermined at intervals with $\frac{\mathrm{n}}{10} \mathrm{KCl}$ and the electrodes replatinised from time to time. The digestion took place in a thermostat kept at $34^{\circ} \mathrm{C}$. The substrate used was 2.5 per cent. solution of sodium caseinogenate to which ammonia was added in the proportion of 1 cubic centimetre of 0.880 ammonia to a litre of the casein solution. Although not more than 0.2 cubic centimetre of serum is required for a determination, it has been the practice in these experiments to use much larger quantities in order, as far as possible, to eliminate errors due to the measurement of very small quantities. It is possible to obtain without difficulty one cubic centimetre of blood from a puncture in the lobe of the ear with the point of a cataract knife, but the majority of the cases investigated have been bled by puncture of the median basilic vein with a small sterilised syringe. The prejudice that exists against this procedure is quite unwarranted. I have personally experienced it about 200 times without the smallest inconvenience or appearance of scarring or thickening over the median basilic. It is perfectly painless when properly done with a sharp needle, and patients generally prefer it to withdrawing blood by ordinary puncture of the finger as being more rapid and less uncomfortable. If the skin is first sterilised and the patient requested to look in another direction the whole operation is conducted without any trouble; the application of dressing afterwards is unnecessary and alarming. It is only necessary to elevate the arm for a few seconds after withdrawing the syringe to prevent any oozing of blood.

The serum should be separated as soon as a firm clot has formed and kept in an ice chest. There is some evidence that the antitryptic power of serum left in contact with the corpuscles diminishes slowly, probably from the setting free of their intracellular enzymes by the disintegrating leucocytes. I have been unable to find any evidence of diminution of antitryptic activity in aseptic serum kept in the cold for many days. As a convenient unit of conductivity the reciprocal megohm or gemmho, as introduced by Waller, may be used. Asepticity is secured by the presence of formalin, 5 cubic centimetres of 5 per cent. formol being added to every litre of the stock casein solution. The formalin itself has a slight inhibitory action on tryptic activity.

In an actual experiment the following technique was observed. The conductivity vessels each received 10 cubic centimetres of casein solution and to this the serum was added. The serum and substrate were now allowed to reach the temperature of the thermostat and an appropriate quantity of trypsin solution added. The trypsin degenerates with such rapidity in a warmed solution that it was deemed expedient to always add the measured pipetteful of trypsin in the cold state, as in the measuring of the ferment into a succession of vessels sufficient time may elapse for an appreciable degeneration of the ferment in the heated state to take place. The vessels are now allowed to stand for ten minutes before the first reading of the electro-conductivity is made, dummy experiments having shown that an amount of fluid at room temperature equal to that of the trypsin used when added to the casein-charged vessel took $7 \cdot 5$ minutes to reach the mean temperature of the thermostat. The readings were afterwards made at regular intervals of 15 minutes. Duplicate vessels were used, so that in every case the mean of two readings was obtained. A point to which importance is attached is the agitation of each vessel while the trypsin solution is being run in from the pipette, otherwise discordant results are obtained which can be accounted for by the possible combination of enzyme and casein molecule before the enzyme has come into contact with the serum as a whole.

The use of trypsin in preference to the proteolytic enzyme of pus cells or leucocytes was only determined upon after a series of experiments comparing the two enzymes which revealed no perceptible difference in the inhibitory action of serum toward the two enzymes. The possibility of a progressive loss of inhibitory power of the serum throughout the course of the reaction is one that has to be considered. Bayliss showed that when sufficient serum was added to a trypsin solution to completely prevent its attacking the caseinogen for at least two days, the caseinogen is slowly attacked and the curve may become a straight line with an angle upwards. He considers this a proof that the antitryptic power of the serum slowly degenerates, as had been previously shown to be the case by Dastre for the antitrypsin of intestinal worms. Using the small quantities of serum that are adopted in these experiments $I$ have never found evidence of such degeneration, but with large amounts of serum which has been first mixed with trypsin and then added to the caseinogen solution curves have occasionally occurred resembling those described by Bayliss. As such curves are not obtained when the serum is first diluted by mixing with the casein, and the trypsin is then added to the casein and serum mixture and the whole shaken, it would appear that we have to deal with a condition of absorption of the trypsin by the serum, which is then slowly liberated, and that this phenomenon does not tend to occur in cases in which the serum is already diluted. The question does not, at any rate, arise in connexion with the ssmall quantities of serum used.

The curves obtained, as shown in Fig. 1, representing the

FIG. 1.

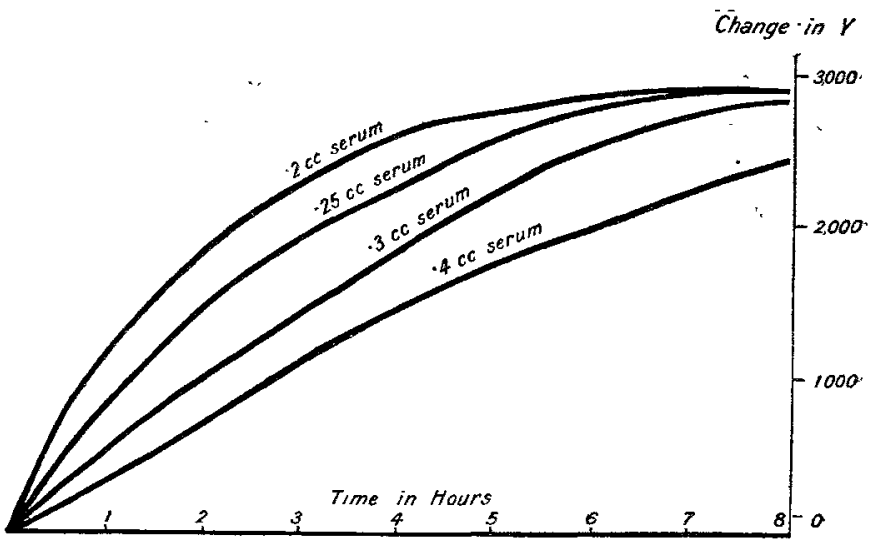

Curve showing rise of conductivity in the casein-trypsin digest after the addition of varying amounts of serum. The vertical figures show the change in gemmbos (or reciprocal megohms) $\gamma$.

FIG. 2.

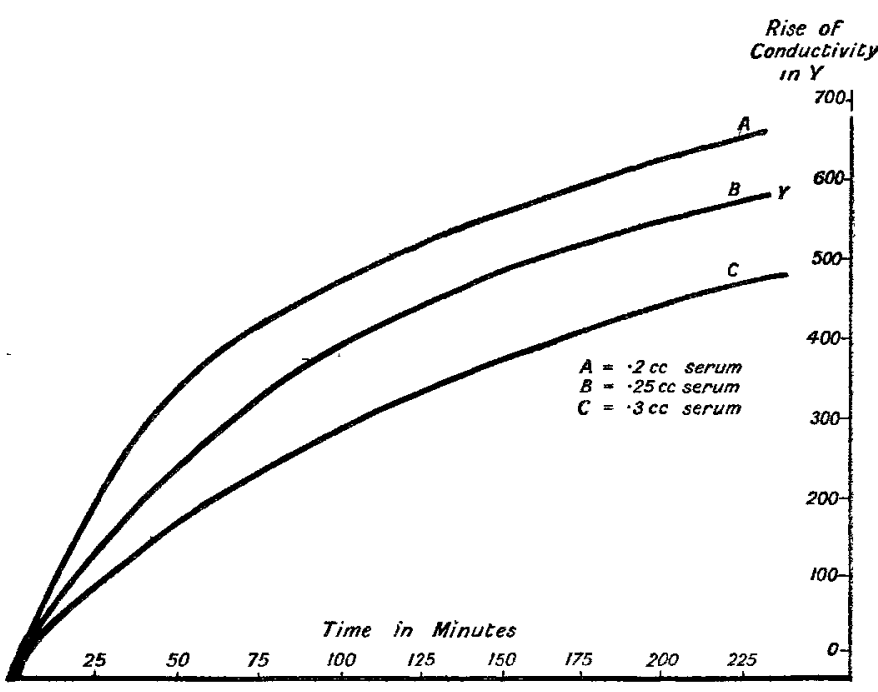

Curve showing direct arithmetical relation of inhibitory power of different amounts of serum in the early stage of the reaction.

quantity of casein converted and time, fall off continuously throughout the experiment from the logarithmic curve, the velocity constant decreasing in value as the end-point is approached. The form of the curve which tends to become 
asymptotic to the base line indicates a tendency towards an equilibrium point with the reaction incomplete. In the initial stages the velocity of reaction appears to be in linear proportion to the amount of enzyme, as pointed out by Bayliss, but this relationship is more and more departed from as the reaction proceeds, so that a stage is finally reached when the velocity of reaction is almost the same for different amounts of enzyme. This latter fact demonstrates the futility of results based on observations of end-point. This tendency towards an equality of rate is due to the diminution of the available amount of substrate but before this can come into play the velocity of reaction has already suffered progressive retardation from the inhibitory action of the accumulating products of digestion. In view of the difficulty of finding a suitable equation for the course of the whole reaction it becomes of importance to prolong the initial stage when the velocities of reaction show a simple linear proportion to the concentration of enzyme as long as possible. This can be done in two fashions-by using the minimal quantity of enzyme possible and using such an amount of substrate that the action of the products of activity does not come into play immediately. In casein solutions such as were used in these experiments it was found that up to a rise of about 550 gemmhos a strictly linear reaction was obtained, and in order to facilitate numerous observations the ferment was reduced as far as possible. It will be seen in the second diagram that a strictly linear proportion is obtained and that the sensibility is such as to show a sufficiently marked variation between additions of 0.05 cubic centimetre of serum. The results below also sufficiently demonstrate the delicacy of the method. To 10 cubic centimetres of casein in four vessels $0.6,0.55,0.5$, and 0.45 cubic centimetres of serum were added and an equal amount of trypsin added to each.

$$
\begin{aligned}
& \begin{array}{llll}
0.6 \text { c.c. } & 0.55 \text { c.c. } & 0.5 \text { c.c. } & 0.45 \text { c.c. } \\
\text { serum. } & \text { serum. } & \text { serum. } & \text { serum }
\end{array} \\
& \text { Rise in } \boldsymbol{\gamma} \text { in } 4 \text { hours }=\begin{array}{llllllllll}
46 & \ldots & \ldots & 53 & \ldots & \ldots & 67 & \ldots & \ldots & 71
\end{array}
\end{aligned}
$$

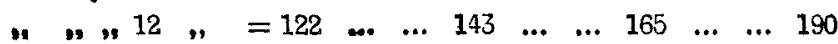

It will be seen by these results that the ordinates representing the rise at any given time give a sufficiently accurate gauge of the relative inhibitory power of three specimens by their direct arithmetical proportion. Inasmuch as it is impossible to obtain the real inhibitory value of the serum since the altered conditions of experiment preclude us from directly comparing the action of enzyme plus serum with enzyme alone, the results have all to be expressed in terms of the normal inhibitory power of an equal amount of serum, and this relation may be called the antitryptic index of the pathological serum examined. Thus if normal serum allows of an increase of conductivity of 250 gemmhos in a given time and Serum $B$ allows of a rise of 200 gemmhos in the same time the antitryptic index of $B$ is said to be $+0 \cdot 25$, whilst if the rise with normal serum be 200 gemmhos and with Serum $C$ the rise of conductivity in equal time reaches 250 gemmhos, the antitryptic power of $\mathbf{C}$ is expressed in the index as $-0 \cdot 25$. Unfortunately, it is impossible to standardise the enzyme added, though in successive experiments, in order to obtain similar conditions, it is well to add an approximately equal amount. It is therefore necessary to have a comparison with normal in each series of experiments. Up to recently this was always done with normal serum obtained from the observer, but the nuisance of undergoing daily punctures may be obviated by use of horse serum kept under aseptic conditions and standardised against normal at intervals of a few days.

It is necessary to assure oneself of the constancy of normal serum. The antitryptic power of serum from six normal individuals has been estimated and found to only show differences within the errors of experiment. Standardising the observers' own serum against horse serum, the human serum being obtained at different periods of the day and after prolonged fasting, no noticeable difference has been detected.

The antitryptic poner of tuberouious serum.-In this preliminary notice it is proposed to deal only with observations carried on with the serum of tuberculous patients. The data which are being obtained with regard to other diseases are not yet sufficiently numerous to allow of any definite statement of results. The patients examined were 25 in number and included cases in my beds at St. George's Hospital, together with those of Dr. A. Latham who very kindly gave me every facility for examining his cases. Some of the more valuable continued observations were done on patients from Mr. E. C. Hort's practice, and it was from a fund under his control for the investigation of serum-therapy that the expenses of this research have been met.

Appended is a table of the results obtained in investiga. tions of the antitryptic index in 25 cases of pulmonary

\begin{tabular}{|c|c|c|c|c|c|c|c|}
\hline \multicolumn{2}{|c|}{ 1. Temperature } & $=$ & $97 \cdot 0^{\circ}$. & .....A & ryp & ndex & +0.65 \\
\hline 2. & ", & $=$ & $98 \cdot 5^{\circ}$ & $\cdots \cdots$ & $"$ & " & +0.2 \\
\hline 3. & ", & $=$ & $98 \cdot 5^{\circ}$ & ...... & ," & " & +0.16 \\
\hline 4. & , & $=$ & $99 \cdot 0^{\circ}$ & ...... & , & ," & Normal. \\
\hline 5. & ", & $=$ & $980^{\circ}$ & ....... & ", & , & +0.2 \\
\hline 6. & , & $=$ & $98.5^{\circ}$ & ...... &, & ", & Normal. \\
\hline 7. & , & $=$ & $100 \cdot 0^{\circ}$ & ....... & , & $"$ & +0.32 \\
\hline 8. &, & $=$ & $99.0^{\circ}$ & $\ldots \ldots$ &, & ", & Normal. \\
\hline 9. & , & $=$ & $98 \cdot 5^{\circ}$ & ....... & ," & ," & -0.09 \\
\hline 10. & ", & $=$ & $100 \cdot 5^{\circ}$ & ...... & ", & ," & +0.2 \\
\hline 11. & ," & $=$ & $98 \cdot 5^{\circ}$ & $\ldots \ldots$ & ", & " & -0.1 \\
\hline 12. & , & $=$ & $99 \cdot 5^{\circ}$ & ...... & ," & ", & Norm: \\
\hline 13. & $"$ & $=$ & $98 \cdot 5^{\circ}$ & ...... & ", & ,. & -0.1 \\
\hline 14. & ", & $=$ & $101 \cdot 0^{\circ}$ & ...... & ", & $" 4$ & +0.05 \\
\hline 15. & ", & $=$ & $99 \cdot 0^{\circ}$ & $\ldots \ldots$ & $"$ & $"$ & +0.15 \\
\hline 16. & , & $=$ & $100 \cdot 0^{\circ}$ & $\ldots \ldots$ & ", & , & +0.07 \\
\hline 17. & , & $=$ & $102 \cdot 0^{\circ}$ & $\ldots \ldots$ & ", & ", & Normal \\
\hline 18. & ", & $=$ & $100 \cdot 0^{\circ}$ & ....... & , & , & +0.07 \\
\hline 19. & ", & $=$ & $98 \cdot 5^{\circ}$ & ....... & $"$ & ", & +0.3 \\
\hline 20. & ,, & $=$ & $99 \cdot 0^{\circ}$ & ...... & "' & $\because$ & +0.5 \\
\hline 21. & ," & $=$ & $100 \cdot 0^{\circ}$ & $\ldots \ldots$ & ", & " & +0.3 \\
\hline 22. & ", & $=$ & $98 \cdot 0^{\circ}$ & $\ldots \ldots$ & , & , & +0.05 \\
\hline 23. & ", & $=$ & $98 \cdot 5^{\circ}$ & $\ldots \ldots$ & ," & ,, & +0.4 \\
\hline 24. & ," & $=$ & $97 \cdot 0^{\circ}$ & $\ldots \ldots$ & ", & , & +0.25 \\
\hline 25. & ", & $=$ & $98 \cdot 5^{\circ}$ & $\ldots \ldots$ & ", & $"$ & $\mathrm{~m}$ \\
\hline
\end{tabular}
tuberculosis :-

It will be obvious that the rise of antitryptic index in these cases has had no direct relation to the temperature. Con sideration of these cases from the clinical point of view gave, however, the impression that the antitryptic index furnishes some indication of the degree of tuberculous intoxication. In the majority of cases that showed signs of progressive improvement the antitryptic index was normal or showed only a slight rise. In those cases in which the disease was progressing the index was much raised. In order to test the accuracy of this view two methods were adopted. Successive observations were made on a number of cases who either showed tendency to improvement or who were becoming markedly worse, and secondly, the response of persons with high or low antitryptic indices towards inoculation with tuberculin was observed by taking the antitryptic power of their serum before and after inoculation.

CASE 1.-A thin alcoholic man, aged 46 years, suffering from extensive tuberculous disease of both lungs. He was first seen at the beginning of September and except for a brief improvement attributable to his improved surroundings he steadily went down hill. Up to the beginning of October he was free from fever but after that he had a continuous irregular temperature ranging between $99^{\circ}$ and $101^{\circ}$ till his death in January of this year. On admission on Sept. 14th the antitryptic index was $+0 \cdot 2$. A week later the patient had shown some slight improvement and the index was +0.16 . On Sept. 28th the patient was given 0.001 gramme of tuberculin by the mouth and the blood taken immediately before and 12 hours after the inoculation. The rise of antitryptic index, taking that of the blood before inoculation as the normal, was $+0 \cdot 2$. The antitryptic index of the blood before inoculation was $+0 \cdot 2$. The patient showed a rise of temperature of half a degree after the inoculation. The patient was treated by inoculations of 0.001 gramme of tuberculin weekly. At the end of October his antitryptic index was +0.4 and the effect of an inoculation was only a rise in the index as compared with the serum before inoculation of +0.05 . In December the index remained about +0.4 and an inoculation gave no response. No furthur inoculations were performed till his death in January. It would appear therefore that as the patient became worse his power of responding to inoculations diminished whilst the antitryptic index increased. It here becomes important to decide whether the diminution of power of response was the effect of a high antitryptic index or the effect of a succession of inoculations. In the four cases here given it will be seen that the evidence points to the inability of a person with a high antitryptic index to 
respond to an inoculation in the same degree as a patient with a low index.

(1) Antitryptic index $=+0 \cdot 5$. Index of serum after inoculation to that before $=+0.07-$

(2) Antitryptic index $=+0.4$. Index of serum after inoculation to that before $=+0.1-$

(3) Antitryptic index $=+0.08$. Index of serum after inoculation to that before $=+0 \cdot 2$

(4) Antitryptic index $=$ Normal. Index of serum after fnoculation to that before $=+0.22$

In each case the inoculation consisted in the administration of 0.001 gramme of tuberculin.

CASE 2.-A young man, aged 23 years, discharged from the army with well-marked tuberculous infiltration of both lungs. This patient, who was under the care of Mr. Hort, reacted well to treatment and after four months was discharged with the disease arrested. Throughout the first two months of treatment he had an irregular temperature, ranging from $99^{\circ}$ to $101^{\circ}$; during the third month the temperature gradually became normal. On admission the index was on the minus side, being -0.01 . Two weeks later it was found to be normal and remained normal till the time of the patient's discharge. It may be noted that throughout the whole course of his treatment he steadily gained in weight. The reaction to inoculation by 0.001 tuberculin was taken on five occasions. On admission the serum after inoculation, as compared with that before, showed a rise of index of +0.5 . The patient was inoculated twice a week for five weeks, at the end of which period an index of +0.06 was obtained. At the end of the third month the postinoculative index was +0.08 . Two further observations gave no rise of index at all towards the end of the patient's treatment.

CASE 3.-A woman suffering from tuberculous involvement of the whole of the left lower lobe gave at the beginning of her treatment an antitryptic index of $+0 \cdot 18$. Examined three months later, when the disease appeared to have been arrested, the incex was normal. In this case no inoculations were performed but the patient had been treated by the method of auto-inoculation by Mr. Hort. Two other cases undergoing cure showed a variation of antitryptic activity in the same direction.

Two cases dying from acute pulmonary tuberculosis were examined shortly before death; in both cases the antitryptic index was high, being +0.4 and +0.5 respectively. In a series of 33 inoculations studied in tuberculous patients a rise of antitryptic index followed the inoculation in 24 cases, and the amount of rise appears to vary inversely to the antitryptic index in the patient as regards normal. The method would appear to afford additional evidence, were any needed, of the efficacy of the oral administration of tuberculous vaccines introduced by $\mathrm{Dr}$. Latham. In four cases in which inoculation was practised on non-tuberculous subjects no rise was noted. Attempts have been made to find the time relations of the post-inoculative rise. The earliest period at which the rise was found was four hours after a subcutaneous inoculation. The index appears to return to its former level in about 40 hours if the dose be not so excessive as to cause marked reaction. In no case was a negative phase noted. Such rises of index after inoculation appear to occur independently of a rise of tem. perature, but in those cases in which no rise was noted there was never any increase of temperature succeeding inoculation.

The method of giving serum by the mouth together with vaccine has been used by Dr. Latham and he has noted that the vaccine can be given in this method in much larger doses. In a case under his care it was found that no rise of index followed an inoculation of ${ }_{30}^{1} \delta$ of tuberculin and on inquiry it was found that the dose had been given with five cubic centimetres of normal horse serum. After a few days the dose was repeated with the serum omitted and on this occasion a rise of index of +4 was noted. In three cases of tuberculous disease of the joints no rise of the antitryptic index was found and results from inoculation proved to be negative. In a case of tuberculous glands operation was followed by a rise of antitryptic index of $+0 \cdot 2$ on the morning after operation.

It is hoped that if further researches confirm and extend the conclusions presented in this preliminary note we may be in possession of an easy physical method which has a negligible experimental error and unlike opsonic determinations does not necessarily remain in the hands of a few highly-skilled observers. By such a method the dosage of inoculation may be surely gauged when the temperature chart affords no help as occurs so frequently in cases of febrile tuberculosis. The index may also prove of prognostic value and the results hitherto arrived at seem to show that it is possible by its means to decide what cases are in need of inoculative treatment.

The materials so far at our disposal do not permit the discussion of the significance of the variations of the antitryptic power of the blood. Whether it be an expression of the attempt on the part of the organism to protect damaged cell tissue from autolysis, as Baer has demonstrated in vitro when serum is added to autolysing tissue, or whether it be an expression of the changes in antitryptic content evoked by the inoculation of the organism with the tryptic ferments from damaged and disintegrating leucocytes, are questions which must be determined by other methods than those of clinical observation. At present work is being carried on to determine the variation that may occur in the antipeptic power of the blood. As Opie and others have shown, both tryptic and peptic ferments occur in leucocytic ferments, and the latter occurs in the lymphocytes alone, whilst in the polymorphonuclear cells the tryptic ferment is predominant. Attempts are also being made to determine the question of the specificity of serum antiferments to the endo-enzymes of different tissues. A line of inquiry that much requires elucidation is one to determine the question as to whether variations of antitryptic power are due to increase or diminution of the antitryptic part of the serum or to variations in amount of the auxylytic substance which is generally supposed to be attached to the globulin moiety. In conclusion, I wish to express my thanks to Dr. Waller, in whose laboratory this work has been conducted, for his kindness in affording me every facility for my experiments.

Bibliography.-Miiller and Jochmann : Münchener Medicinische Wochenschrift, 1906, No. 4. For references to further literature see Miiller, Deutsches Arehiv für Klinische Medicin, 1907, Band 91-3. Bittorf: Ibid., vol. xci., No. 1-2. Wiens: Ibid., vol. xci,, No. 5-6 Münchener Medicinische Wochenschrift, 1907, No. 53. Brieger and Trebing : Berliner Klinische Wochenschrift, 1908, No. 22. Bergmann and Meyer: Ibid., 1908, No. 37. Bayliss: Archives des Sciences Biologiques, 1904, p. 261.

\section{SPONTANEOUS CURE OF THORACIC ANEURYSM.}

\section{By SIR THOMAS OLIVER, M.D. GLASG., LL.D.,} D.SC. SHEFF., F.R.C.P. LoND.,

PROFESSOR OF PHYSIOLOGY IN THE COLLEGE OF MEDICINE, AND PHYSICIAN TO THE ROYAI VICTORIA INFIRMARY, NEWCASTLE-UPON-TYNE.

LATENT thoracic aneurysm unattended by symptoms and physical signs, yet suddenly rupturing and causing immediate death, is not unknown, but with the spontaneous cure of thoracic aneurysm many of us are not familiar. As the possibility of such an occurrence is even questioned by some physicians I may be excused for bringing the following case under the notice of the readers of THE LANCET. The case was that of a man who, although the subject of recurrent dyspnoea especially of a nocturnal character, presented on examination no physical signs of disease of the aorta. In him the difficulty of breathing took the type of renal asthma and was relieved by the treatment usually adopted for it. At this stage in the history of the case and with all the details of the necropsy before me there seems to be nothing to alter in regard to what has been stated as to the cause of the shortness of breath.

The patient, a man aged 55 years, was admitted into the Royal Victoria Infirmary, Newcastle-upon-Tyne, on April 15th, 1908, suffering from extreme weakness and difficulty of breathing. Seven years ago he was an in-patient of the Royal Infirmary, Liverpool, on account of acute rheumatism from which he had suffered for four years previously. 12 years ago, while employed in marine works in Philadelphia, U.S.A., he lost his wife and four children all within 48 hours from malarial fever, the disease being epidemic in the city at the time. Four years ago the patient was suddenly seized with vomiting and diarrhœa. The 